\title{
BMJ Open Analysis of eGFR index category and annual eGFR slope association with adverse clinical outcomes using real- world Japanese data: a retrospective database study
}

\author{
Ling Zhang (D) , ${ }^{1}$ Sibylle Hauske, ${ }^{2,3}$ Yasuhisa Ono, ${ }^{4}$ Moe H Kyaw, ${ }^{1}$ \\ Dominik Steubl, ${ }^{2,5}$ Yusuke Naito, ${ }^{6}$ Keizo Kanasaki (D) ${ }^{7,8}$
}

To cite: Zhang L, Hauske $\mathrm{S}$, Ono Y, et al. Analysis of eGFR index category and annual eGFR slope association with adverse clinical outcomes using real-world Japanese data: a retrospective database study. BMJ Open 2022;12:e052246. doi:10.1136/ bmjopen-2021-052246

- Prepublication history and additional supplemental material for this paper are available online. To view these files, please visit the journal online (http://dx.doi.org/10.1136/ bmjopen-2021-052246).

$\mathrm{LZ}, \mathrm{SH}$ and $\mathrm{YO}$ contributed equally.

Received 21 April 2021 Accepted 26 January 2022

D Check for updates

(c) Author(s) (or their employer(s)) 2022. Re-use permitted under CC BY-NC. No commercial re-use. See rights and permissions. Published by BMJ.

For numbered affiliations see end of article.

Correspondence to Ling Zhang;

ling_3.zhang@boehringeringelheim.com

\section{ABSTRACT}

Objectives Real-world clinical outcome data of patients with an above-normal estimated glomerular filtration rate (eGFR) and increasing eGFR over time (eGFR slope) are scarce. Although eGFR is commonly recorded, eGFR slopes are rarely used for adverse outcome risk categorisation in clinical practice. We investigated the association of above-normal/below-normal eGFR ranges and increasing/ declining eGFR slopes with clinical outcomes in Japan. Design Observational cohort study.

Setting Primary and acute care hospitals; 423 centres. Participants 57452 patients aged $\geq 16$ years with $\geq 3$ eGFR values (latest available January 2013-December 2016) from the Japanese Medical Data Vision database were stratified into six index eGFR and six eGFR slope groups (slopes calculated using a linear mixed model). Primary and secondary outcome measures Timeto-event analyses of cardiovascular mortality, all-cause mortality (ACM), all-cause hospitalisation (ACH) and cardiovascular and major kidney events. eGFR and slope groups were analysed by Cox proportional hazard models with multivariable adjustment, using normal eGFR/little-tono slope groups as reference.

Results Higher risk of clinical outcomes was observed with declining eGFR slope groups versus the reference group; the HR $(95 \% \mathrm{Cl})$ for slope $\leq-5 \mathrm{~mL} / \mathrm{min} / 1.73 \mathrm{~m}^{2} /$ year: cardiovascular events 1.8 (1.4 to 2.2), ACH 1.8 (1.5 to 2.1), and ACM 2.8 (1.9 to 4.2) and was non-significant for kidney events 1.5 ( 0.9 to 2.5). A similar, but nonsignificant, pattern was observed with increasing slope groups (slope $>3 \mathrm{~mL} / \mathrm{min} / 1.73 \mathrm{~m}^{2} /$ year HR $(95 \% \mathrm{Cl}$ ): cardiovascular events 1.2 (0.9 to 1.5), ACH 1.1 (0.9 to 1.4) and ACM 1.5 (0.9 to 2.3)).

Above-normal and below-normal eGFR groups were associated with poorer outcomes versus the reference group, but kidney events were associated with belownormal eGFR only.

Conclusion Poorer clinical outcomes were observed not only for below-normal eGFR and declining eGFR slope groups but also for certain above-normal eGFR and increasing slope groups. eGFR and eGFR slope may, therefore, be useful for identifying patients at high risk of adverse clinical outcomes.

\section{Strengths and limitations of this study}

- This study included a large number of patients (57 452) with estimated glomerular filtration rate (eGFR) data gathered in a real-world setting, which was not restricted to a specific high-risk patient population.

- Due to the inclusion of the most recent data available in the Japanese Medical Data Vision database (study period: 1 January 2012 to 31 August 2019), our findings are likely to reflect the current patient population and, therefore, also reliable and informative for use in guiding current clinical recommendations.

- The association of both eGFR slope and eGFR index groups with adverse clinical outcomes was determined using multiple statistical models (crude, multivariable and 'full' Cox proportional hazard models), which is supportive of the robustness of the risk estimations.

- Due to the nature of the database used, which has a disproportionately high number of oncology patients compared with the overall population and mortality data based on inpatient deaths only, the generalisability of our findings to the wider Japanese population is unknown.

\section{INTRODUCTION}

The association between reduced kidney function, as measured by below-normal estimated glomerular filtration rate (eGFR), and increased risk of adverse clinical outcomes is well known, including all-cause mortality (ACM), cardiovascular (CV) mortality and end-stage kidney disease. ${ }^{12}$

Decline of GFR is characteristic of kidney disease progression to kidney failure. Furthermore, epidemiological data have suggested that declining eGFR over time (ie, eGFR slope) is associated with increased risk of adverse clinical outcomes, such as $\mathrm{CV}$ disease, mortality ${ }^{3}$ and kidney outcomes. ${ }^{4}$ Despite growing evidence that eGFR slopes 
might be a reliably predictive surrogate end point of kidney outcomes, they are not yet widely used in this way in clinical trials. ${ }^{45}$ The use of eGFR slopes in clinical trials has become a field of ongoing development since publication of the outcomes of a 2018 scientific workshop, sponsored by the National Kidney Foundation in collaboration with the US Food and Drug Administration and European Medicines Agency. ${ }^{6}$ The workshop collaborators concluded that GFR slopes fulfil the criteria for use as a surrogate end point for chronic kidney disease (CKD) progression in clinical trials under certain conditions; however, currently, regulatory acceptance based on eGFR slopes as a surrogate end point remains challenging and is limited to rare kidney diseases. ${ }^{78}$

A recent follow-up post hoc analysis of cohorts from a multinational randomised controlled trial of patients with type 2 diabetes (T2D) found an association between declining eGFR slope and elevated risk of a composite outcome of major kidney events, major macrovascular events and ACM. ${ }^{9}$ It has also been shown that declining eGFR slope is associated with a higher risk of kidney outcomes in the Japanese population. ${ }^{10}$ However, unlike other countries such as Canada and Belgium, ${ }^{11}{ }^{12}$ data that support an association between eGFR slopes and $\mathrm{CV}$ or hospitalisation risk and include above-normal and below-normal eGFR ranges are currently scarce for the Japanese population.

There is also a paucity of evidence regarding the association between above-normal eGFR and risk of clinical outcomes. Notably, cardiokidney outcomes trials in patients with CKD, with or without T2D, commonly exclude patients with above-normal eGFR values, ${ }^{13-18}$ and so are limited to selected, highrisk groups (mainly defined by normal-to-low eGFR ranges mostly with presence of proteinuria). To our knowledge, no data from clinical trials are currently available regarding the relationship between clinical outcomes and absolute eGFR or eGFR slope across the full spectrum of kidney function with a wide variety of comorbidities. Additionally, little is known regarding the real-world clinical outcomes and prognosis of Japanese patients with above-normal eGFR ranges and increasing eGFR slopes. Therefore, in order to fill this knowledge gap, we investigated whether both above-normal and below-normal eGFR as well as both increasing and decreasing eGFR slopes are associated with increased risk of clinical outcomes in the Japanese population.

Furthermore, despite the availability of existing eGFR data in electronic health records, eGFR slopes are rarely used in a structured way to evaluate the risk of individual patients for adverse CV and kidney outcomes in clinical practice. In order to determine whether existing eGFR data may be of value for the identification of patients at high risk of adverse clinical outcomes in the Japanese population, we assessed the association between eGFR data and the risk of clinical outcomes using the Japanese Medical Data Vision (MDV) database.

\section{MATERIALS AND METHODS}

\section{Study design and data source}

Anonymised data from the MDV database, Japan's largest commercially available administrative diagnosis procedure combination database (http://www.mdv.co.jp), were used in this retrospective, observational cohort study. As of September 2020, the MDV database contained data from nearly 33 million inpatients and outpatients treated at any of 423 hospitals (covering approximately $24 \%$ of acute hospitals in Japan). The MDV database holds patient information including demographics, diagnoses, medical procedures, medical costs and prescriptions. Over $80 \%$ of patient data are collected from outpatient settings. The database population has an age and sex distribution similar to the Japanese population receiving healthcare services in either inpatient or outpatient settings.

Patients $\geq 16$ years of age and with three or more available eGFR values, with the latest eGFR value available between 1 January 2013 and 31 December 2016, were included in this study. The study period was from 1 January 2012 to 31 August 2019, that is, the end of database coverage at the time of the study. The baseline period used was 1 year prior to the index date, and the follow-up time was continued from the index date until the latest record in the database. Index eGFR was defined as the last eGFR value within the selection period (1 January 2013 to 31 December 2016). eGFR slope was calculated based on eGFR measurements during the selection period. Follow-up started immediately after the last eGFR measurement used to calculate the slope (ie, the index date). Exclusion criteria included patients who underwent dialysis (defined using the International Classification of Diseases, Tenth Revision (ICD-10) codes J038, C102-2, J042, C102, C155, K635-3) or experienced kidney failure (N17.0-17.9 or N18.4-18.6) during the baseline period, those with a diagnosis of CV (I00-09, I11, I13, I20-I51, I10, I12, I15 or I60-69) and kidney outcome events (end-stage renal disease diagnosis (ESRD) code N18.5, dialysis procedure codes J038, C102-2, J042, C102 or C155 or renal transplant procedure codes Z94.0, T86.1 or K780-2) during the baseline period and patients with acute kidney injury (AKI, N17) or kidney failure (presence of diagnosis code of ESRD, dialysis procedure codes or renal transplant codes as above) during the selection period before the index date (figure 1).

\section{Outcomes and kidney transplant codes}

The primary outcomes of the study were CV death and ACM (based on inpatient death data). The secondary outcomes were all-cause hospitalisation $(\mathrm{ACH})$ and hospitalisation due to any CV event (defined as any stroke, chronic heart failure or myocardial infarction event) or any major kidney event (defined as any ESRD, including any dialysis treatment, kidney transplantation or AKI). Kidney events included end-stage kidney disease (ICD-10 code: N18.5) dialysis procedure (procedure codes: J038, C102-2, J042, C102, C155 or K635-3), kidney transplant (procedure codes: Z94.0, T86.1 or K780-2) and acute 


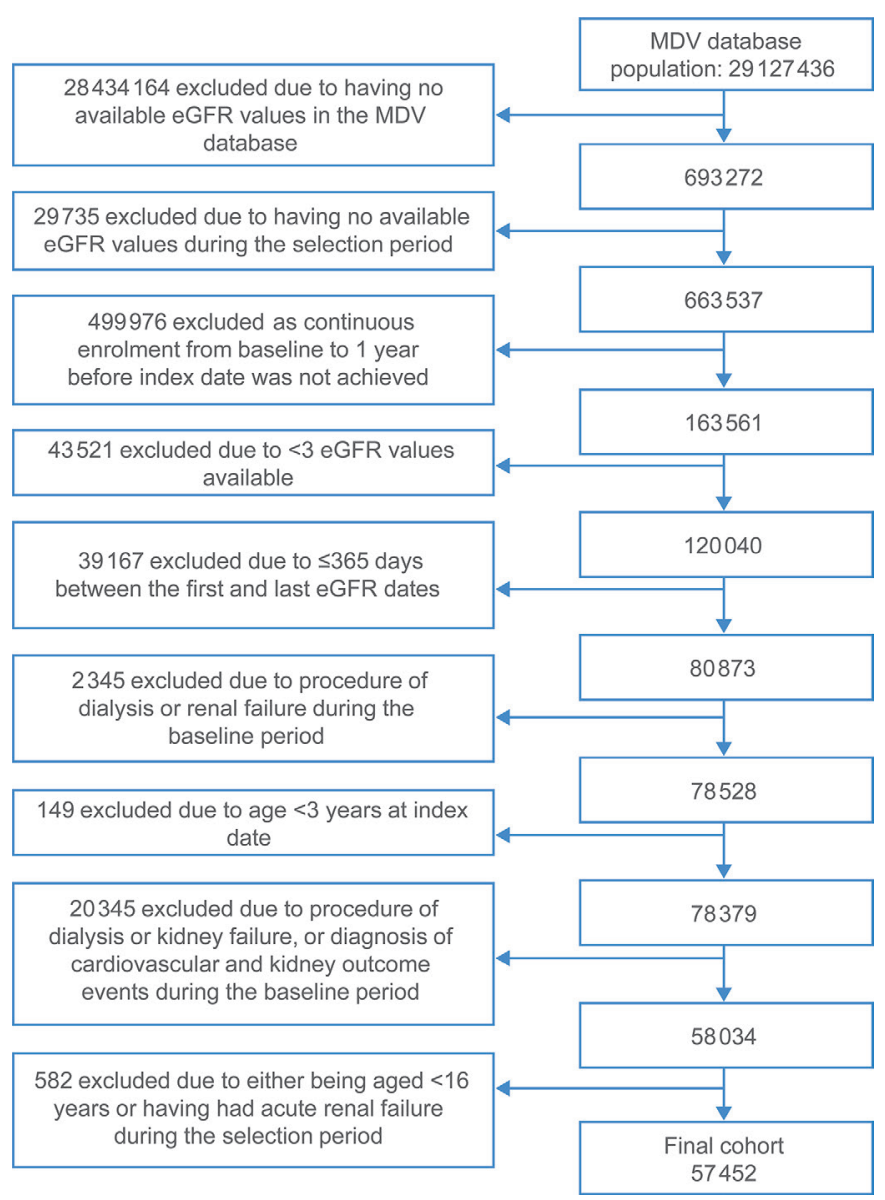

Figure 1 Cohort selection. eGFR, estimated glomerular filtration rate; MDV, Medical Data Vision.

kidney failure (ICD-10 code: N17). Outcomes were identified using ICD-10 codes as well as MDV-specific procedure codes and medication codes by Anatomical Therapeutic Chemical (ATC) system.

\section{Statistical methods}

eGFR was calculated using the formula: eGFR $\left(\mathrm{mL} / \mathrm{min} / 1.73 \quad \mathrm{~m}^{2}\right)=194 \times(\text { serum creatinine })^{-1.094} \times$ (age $)^{-0.287}(\times 0.739$ if female). For index eGFR analyses, patients were stratified into six index eGFR categories: $\geq 60$ to $<90$ (category 1 , reference), $<15, \geq 15$ to $<30, \geq 30$ to $<60, \geq 90$ to $<120, \geq 120$ to $\leq 200 \mathrm{~mL} / \mathrm{min} / 1.73 \mathrm{~m}^{2}$

\begin{tabular}{lll} 
Table 1 & Index eGFR and eGFR slope category definitions \\
\hline Category & $\begin{array}{l}\text { Index eGFR, } \mathbf{m L} / \\
\text { min/1.73 } \mathbf{~ m}^{2}\end{array}$ & $\begin{array}{l}\text { eGFR slope, } \mathbf{m L} / \mathbf{m i n} / \mathbf{1 . 7 3} \\
\mathbf{m}^{2} / \text { year }\end{array}$ \\
\hline 1 (reference) & $\geq 60$ to $<90$ & $>-1$ to $\leq 1$ \\
2 & $<15$ & $\leq-5$ (rapid eGFRdec group) \\
3 & $\geq 15$ to $<30$ & $>-5$ to $\leq-3$ \\
4 & $\geq 30$ to $<60$ & $>-3$ to $\leq-1$ \\
5 & $\geq 90$ to $<120$ & $>1$ to $\leq 3$ \\
6 & $\geq 120$ to $\leq 200$ & $>3$ (rapid eGFRinc group) \\
\hline
\end{tabular}

eGFR, estimated glomerular filtration rate; eGFRdec, eGFR decliner; eGFRinc, eGFR increaser. (table 1). For eGFR slope analyses, patients were stratified into six eGFR slope categories: $>-1$ to $\leq 1$ (category 1 , reference), $\leq-5$ (rapid eGFR decliner (eGFRdec) group), $>-5$ to $\leq-3,>-3$ to $\leq-1,>1$ to $\leq 3,>3 \mathrm{~mL} / \mathrm{min} / 1.73 \mathrm{~m}^{2}$ / year (rapid eGFR increaser (eGFRinc) group) (table 1).

A linear mixed model was used to calculate annual eGFR slope, which included least squares regression as a fixed effect and patient as a random effect. Cox proportional hazard models were applied to index eGFR and eGFR slope categories 2-6, with category 1 as the reference group, with time-to-event analyses conducted for each outcome to generate HRs. The 'crude model' included only slope categories or index eGFR categories. The 'multivariable model' for eGFR slope category analyses was adjusted for age, sex, medical history such as CV disease, solid tumours and leukaemia/lymphoma (see table 2), baseline T2D status, baseline Elixhauser Comorbidity Index (ECI) score, baseline medication use and index eGFR value. The 'full model' for eGFR slope included all of these in addition to an interaction term for eGFR slope and index eGFR. The 'multivariable model' for the index eGFR category analyses was adjusted for age, sex, medical history, baseline T2D status, baseline ECI score and baseline medication use. By grouping patients into various cohorts based on eGFR slope, these models reduce potential sources of selection bias and/or channelling bias.

Sensitivity analyses, using a stepwise selection Cox proportional hazard model for covariates, were used to investigate the risk profiles for each of the outcomes investigated in this study.

Patients were stratified into the following subgroups, with time-to-event analyses conducted by eGFR slope and index eGFR category: the presence or absence of tumour (defined using the ICD-10 codes C00-D49) and T2D at baseline (ICD-10 codes E11 or E14). These analyses attempt to address potential selection bias based on comorbidities by checking for consistency of results among population subgroups.

For index eGFR and eGFR slope, patients with missing data were not included. As clinical outcomes were based on hospital data, this data may only capture treated events. However, as all measured outcomes in this study are relatively severe conditions that require treatment, the number of missing values are likely to be very low.

\section{Patient and public involvement}

Patients and/or the public were not involved in the design, conduct, reporting or dissemination plans of this research.

\section{RESULTS}

\section{Patient demographics}

Of 29127436 patients with data available in the MDV database at the time of the study, 57452 patients were eligible for analysis (figure 1). The median follow-up time was 2.4 years, and patients had a median of 10 available 
Table 2 Baseline demographics by eGFR slope category

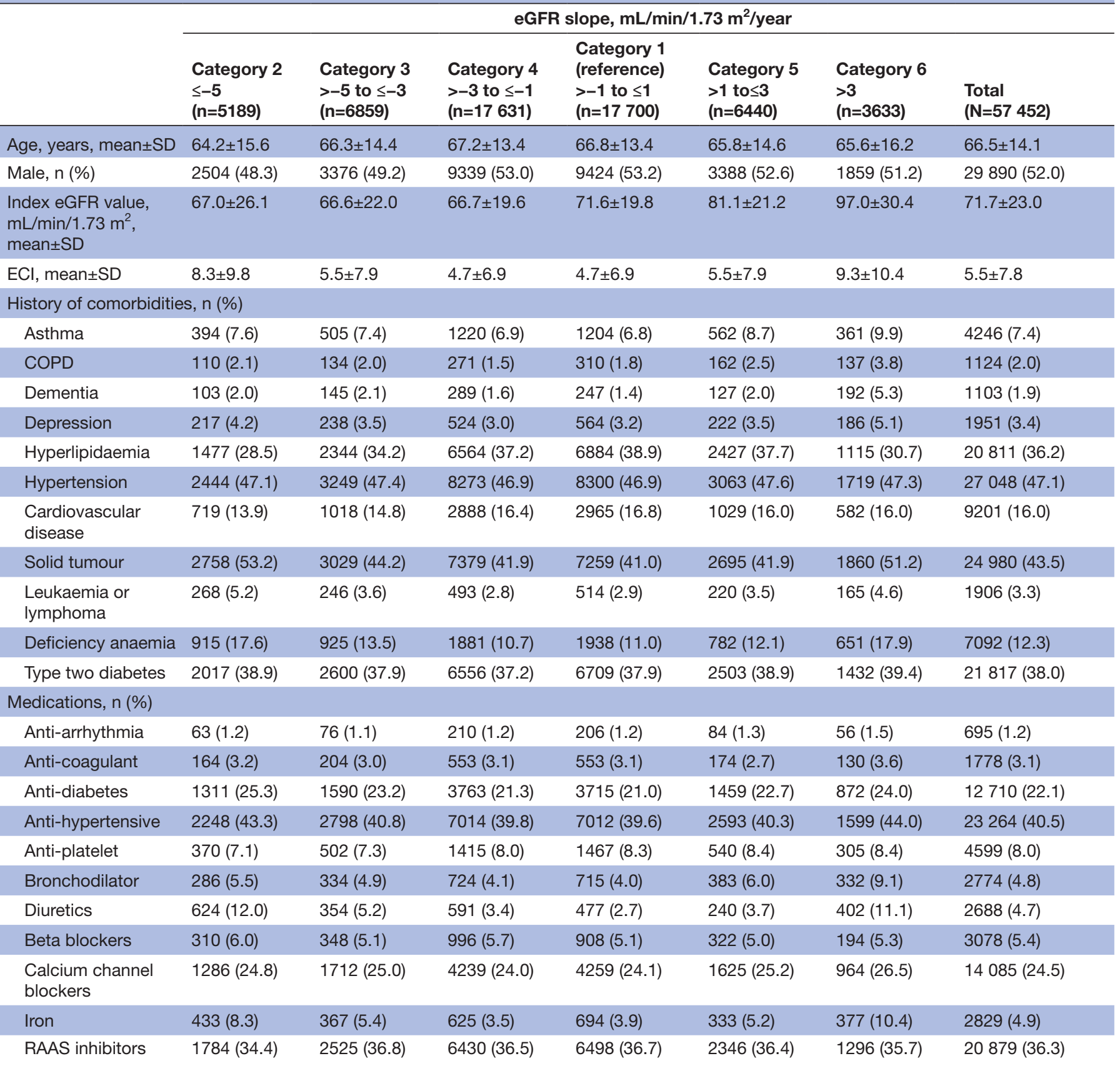

COPD, chronic obstructive pulmonary disease; ECI, Elixhauser Comorbidity Index; eGFR, estimated glomerular filtration rate; RAAS, reninangiotensin-aldosterone system.;

eGFR values. Overall, the mean (SD age was 66.5 years (14.1), 52\% were men and the mean (SD) index eGFR value was $71.7(23.0) \mathrm{mL} / \mathrm{min} / 1.73 \mathrm{~m}^{2}$. There was a high prevalence of hypertension $(47.1 \%)$, solid tumours $(43.5 \%)$ and T2D $(38.0 \%)$ in the study cohort (table 2$)$.

With respect to eGFR slope stratification, the majority of patients (17 700, 61\%) had little-to-no or slowly increasing eGFR slopes (>-1 to $\leq 1 \mathrm{~mL} / \mathrm{min} / 1.73 \mathrm{~m}^{2}$ / year; ie, the reference category) (table 2). The majority of patients $(30942,54 \%)$ were stratified into the index eGFR reference category, which represents the normal eGFR range ( $\geq 60$ to $<90 \mathrm{~mL} / \mathrm{min} / 1.73 \mathrm{~m}^{2}$ ). The lowest
eGFR range $\left(<15 \mathrm{~mL} / \mathrm{min} / 1.73 \mathrm{~m}^{2}\right)$ included the fewest patients $(542,1 \%)$ (table 3$)$.

Overall, higher incidence of comorbidities and higher use of medications at baseline were observed in eGFR slope groups with decline of $\leq-3 \mathrm{~mL} / \mathrm{min} / 1.73$ $\mathrm{m}^{2} /$ year and eGFRinc groups compared with the reference category. In particular, in the slope groups with the highest increase or decrease, a higher proportion of patients had a history of tumours, depression and anaemia and a higher ECI score than in the reference category (table 2). 
Table 3 Baseline demographics by index eGFR category

\begin{tabular}{|c|c|c|c|c|c|c|c|}
\hline & \multicolumn{7}{|c|}{ Index eGFR, mL/min/1.73 m² } \\
\hline & $\begin{array}{l}\text { Category } 2 \\
<15 \\
(n=542)\end{array}$ & $\begin{array}{l}\text { Category } 3 \\
\geq 15 \text { to }<30 \\
(n=1243)\end{array}$ & $\begin{array}{l}\text { Category } 4 \\
\geq 30 \text { to }<60 \\
(n=14336)\end{array}$ & $\begin{array}{l}\text { Category } 1 \\
\text { (reference) } \\
\geq 60 \text { to<90 } \\
(n=30942)\end{array}$ & $\begin{array}{l}\text { Category } 5 \\
\geq 90 \text { to }<120 \\
(n=8935)\end{array}$ & $\begin{array}{l}\text { Category } 6 \\
\geq 120 \text { to } \leq 200 \\
(n=1454)\end{array}$ & $\begin{array}{l}\text { Total } \\
\text { (N=57 452) }\end{array}$ \\
\hline Age, years, mean $\pm S D$ & $68.5 \pm 12.5$ & $74.2 \pm 12.0$ & $73.5 \pm 10.6$ & $66.1 \pm 12.6$ & $57.5 \pm 15.8$ & $51.9 \pm 19.5$ & $66.5 \pm 14.1$ \\
\hline Male, n (\%) & $327(60.3)$ & $678(54.6)$ & $7772(54.2)$ & $16063(51.9)$ & 4425 (49.5) & $625(43.0)$ & $29890(52.0)$ \\
\hline $\mathrm{ECI}$, mean $\pm \mathrm{SD}$ & $11.7 \pm 8.3$ & $9.6 \pm 9.3$ & $5.9 \pm 8.2$ & $5.0 \pm 7.3$ & $5.4 \pm 8.0$ & $7.2 \pm 9.9$ & $5.5 \pm 7.8$ \\
\hline \multicolumn{8}{|c|}{ History of comorbidities, n (\%) } \\
\hline Asthma & $45(8.3)$ & $91(7.3)$ & $1080(7.5)$ & $2232(7.2)$ & $659(7.4)$ & $139(9.6)$ & $4246(7.4)$ \\
\hline COPD & $5(0.9)$ & $28(2.3)$ & $321(2.2)$ & $565(1.8)$ & $165(1.9)$ & $40(2.8)$ & $1124(2.0)$ \\
\hline Hypertension & $437(80.6)$ & $939(75.5)$ & $8536(59.5)$ & $13507(43.7)$ & $3180(35.6)$ & $449(30.9)$ & $27048(47.1)$ \\
\hline $\begin{array}{l}\text { Cardiovascular } \\
\text { disease }\end{array}$ & $174(32.1)$ & $320(25.7)$ & $3192(22.3)$ & $4506(14.6)$ & $891(10.0)$ & $118(8.1)$ & $9201(16.0)$ \\
\hline Solid tumour & $166(30.6)$ & $475(38.2)$ & $6323(44.1)$ & $13612(44.0)$ & $3816(42.7)$ & $588(40.4)$ & $24980(43.5)$ \\
\hline $\begin{array}{l}\text { Leukaemia or } \\
\text { lymphoma }\end{array}$ & $14(2.6)$ & $47(3.8)$ & $513(3.6)$ & $988(3.2)$ & $302(3.4)$ & $42(2.9)$ & $1906(3.3)$ \\
\hline Deficiency anaemia & $230(42.4)$ & 301 (24.2) & 1805 (12.6) & $3329(10.8)$ & $1161(13.0)$ & 266 (18.3) & 7092 (12.3) \\
\hline Type 2 diabetes & $244(45.0)$ & $618(49.7)$ & $5922(41.3)$ & $11342(36.7)$ & $3140(35.1)$ & $551(37.9)$ & $21817(38.0)$ \\
\hline \multicolumn{8}{|l|}{ Medications, n (\%) } \\
\hline Diuretics & $142(26.2)$ & 255 (20.5) & $984(6.9)$ & $915(3.0)$ & 315 (3.5) & $77(5.3)$ & $2688(4.7)$ \\
\hline Beta blockers & $74(13.7)$ & 119 (9.6) & $1089(7.6)$ & $1435(4.6)$ & 315 (3.5) & $46(3.2)$ & $3078(5.4)$ \\
\hline $\begin{array}{l}\text { Calcium channel } \\
\text { blockers }\end{array}$ & $309(57.0)$ & 595 (47.9) & $4434(30.9)$ & 6875 (22.2) & $1644(18.4)$ & $228(15.7)$ & $14085(24.5)$ \\
\hline Iron & $126(23.3)$ & $129(10.4)$ & $734(5.1)$ & $1160(3.8)$ & $533(6.0)$ & $147(10.1)$ & $2829(4.9)$ \\
\hline RAAS inhibitors & $364(67.2)$ & $754(60.7)$ & $6611(46.1)$ & $10355(33.5)$ & $2464(27.6)$ & $331(22.8)$ & 20879 (36.3) \\
\hline
\end{tabular}

COPD, chronic obstructive pulmonary disease; ECI, Elixhauser Comorbidity Index; eGFR, estimated glomerular filtration rate; RAAS, reninangiotensin-aldosterone system.;

Patients with below-normal index eGFR values were more often men and were older than patients with normal and above-normal index eGFR values (table 3). Overall, a higher incidence of comorbidities and higher use of most medications at baseline were observed in the categories with below-normal index eGFR values compared with those in the reference group. Higher use of diuretics and iron medications were seen in those with both belownormal and above-normal index eGFR values compared with those in the normal range.

\section{Risk of major clinical outcomes by EGFR slope category}

Of the 57452 patients included in the analysis, there were 28288 with an event, of which 1411 (5.0\%) were CV death, 2320 (8.2\%) were ACM events, 15103 (53.4\%) were ACH, 8927 (31.6\%) were any CV event and 527 $(1.9 \%)$ were any kidney event.

Using the full model, which includes an interaction term for index eGFR and eGFR slope, the risk of major clinical outcomes was increased in eGFRdec groups compared with the reference group. In particular, the 


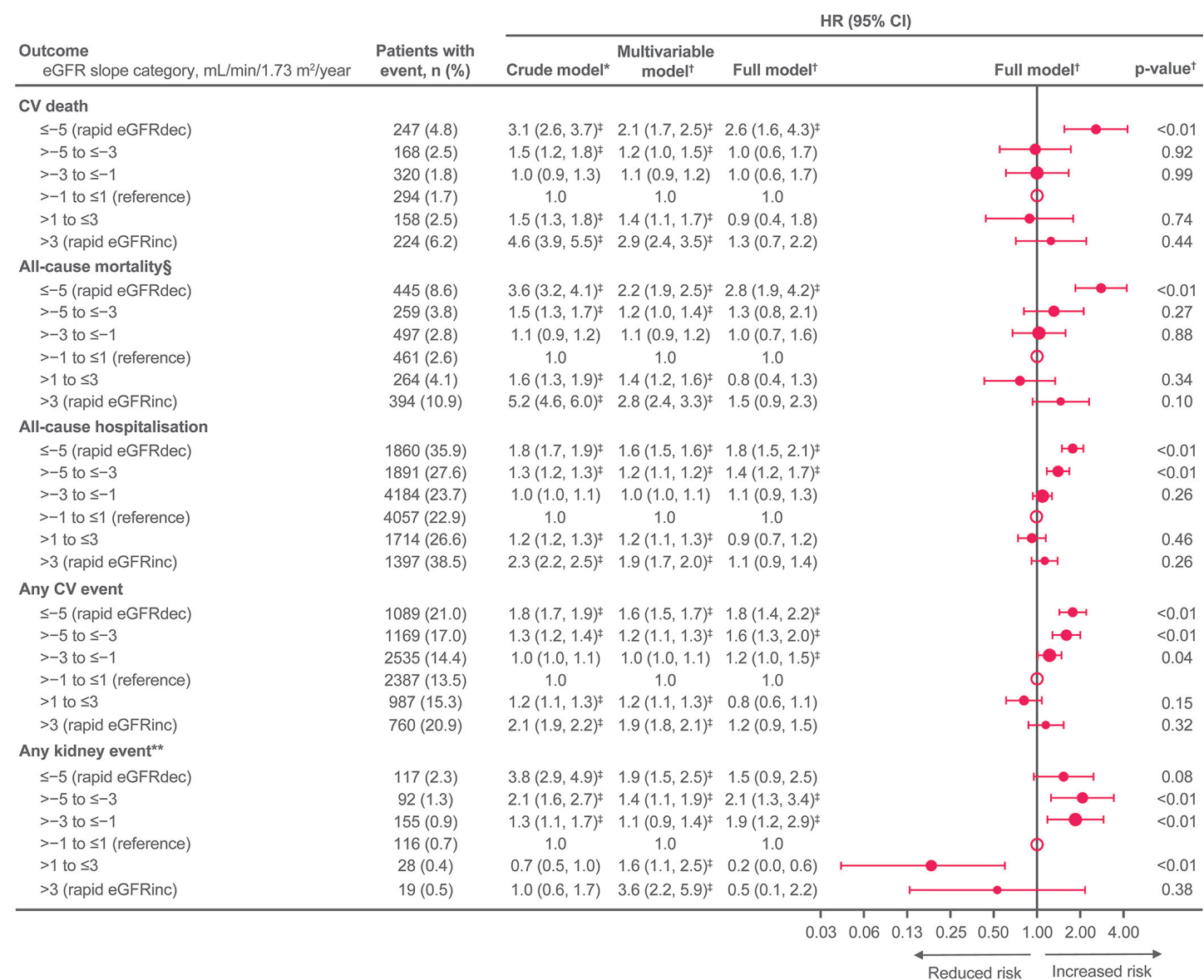

Figure 2 Risk of CV, mortality, hospitalisation and kidney outcomes by eGFR slope category. ${ }^{*}$ Cox proportional hazard model for time-to-event, with outcome and slope categories as independent variables. †Cox proportional hazard model for time-toevent, adjusted for age, sex, medical history, BL type 2 diabetes status, BL ECI score, BL medication use and index eGFR value for the multivariate model, plus interaction terms for slope categories in the full model. Categories 2-6 (eGFR slopes: $\leq-5$, $>-5$ to $\leq-3,>-3$ to $\leq-1,>1$ to $\leq 3,>3$ ) were analysed by outcome, with Category 1 (slope $>-1$ to $\leq 1$ ) as reference. $\ddagger$ Statistically significant HRs. §Based on inpatient death data. ๆStroke, chronic heart failure or myocardial infarction. ${ }^{\star *}$ End-stage kidney disease, dialysis, acute kidney failure or kidney transplant. BL, baseline; CV, cardiovascular; ECI, Elixhauser Comorbidity Index; eGFR, estimated glomerular filtration rate; eGFRdec, eGFR decliner; eGFRinc, eGFR increaser; HR, hazard ratio.

rapid eGFRdec group showed an increased risk of $\mathrm{CV}$ death (HR (95\% CI)) (2.6 (1.6 to 4.3), p<0.01), ACM (2.8 (1.9 to 4.2), $\mathrm{p}<0.01)$, ACH (1.8 (1.5 to 2.1), $\mathrm{p}<0.01$ ) and any CV event (1.8 (1.4 to 2.2), $\mathrm{p}<0.01$ ) (figure 2). In general, the risk of any kidney event was increased in all eGFRdec groups compared with the reference group; however, the increase was non-significant in the rapid eGFRdec group (HR (95\% CI)) (1.5 (0.9 to 2.5$) \mathrm{p}=0.08)$ (figure 2).

For the rapid eGFRinc group, the crude model showed a significantly increased risk of CV outcomes, mortality and hospitalisation compared with the reference category: HR (95\% CI) 4.6 (3.9 to 5.5) for CV death, 5.2 (4.6 to 6.0) for ACM, 2.3 (2.2 to 2.5) for ACH, and 2.1 (1.9 to 2.2) for any CV event. Likewise, a significantly increased risk of these outcomes was observed in the multivariable model: HR (95\% CI) 2.9 (2.4 to 3.5) for CV death, 2.8 (2.4 to 3.3) for ACM, 1.8 (1.7 to 2.0) for ACH and 1.9 (1.8 to 2.1) for any CV event.

In the full model, there was also a trend, though nonsignificant, for higher risk of CV outcomes, mortality and hospitalisation in eGFRinc groups. In the rapid eGFRinc group, the HR (95\% CI) was 1.3 (0.7 to 2.2$), \mathrm{p}=0.44$, for CV death; 1.5 (0.9 to 2.3), $\mathrm{p}=0.10$, for ACM; 1.1 (0.9 to 1.4), $\mathrm{p}=0.26$, for $\mathrm{ACH}$; and 1.2 (0.9 to 1.5 ), $\mathrm{p}=0.32$, for any $\mathrm{CV}$ event (figure 2).

\section{Risk of major clinical outcomes by index eGFR category}

A higher risk versus the reference group $(\geq 60$ to $<90 \mathrm{~mL}$ / $\min / 1.73 \mathrm{~m}^{2}$ ) was observed in below-normal index eGFR categories for major clinical outcomes: in the $<15 \mathrm{~mL} /$ $\min / 1.73 \mathrm{~m}^{2}$ category, the HR $(95 \% \mathrm{CI})$ was 3.2 (2.8 to 3.6), $\mathrm{p}<0.01$, for any CV event; 2.9 (2.2 to 3.9), $\mathrm{p}<0.01$, for CV death; 2.3 (2.0 to 2.6), $\mathrm{p}<0.01$, for $\mathrm{ACH}$; and 2.7 (2.1 to 3.5), $\mathrm{p}<0.01$, for ACM (figure 3). Likewise, in the severely increased eGFR category $(\geq 120$ to $\leq 200 \mathrm{~mL} /$ $\left.\min / 1.73 \mathrm{~m}^{2}\right)$ versus the reference group, the HR $(95 \%$ CI) using the multivariable model was 1.9 (1.6 to 2.2), $\mathrm{p}<0.01$, for any CV event; 2.6 (2.0 to 3.5), $\mathrm{p}<0.01$, for $\mathrm{CV}$ death; 2.2 (2.0 to 2.4), $\mathrm{p}<0.01$, for $\mathrm{ACH}$; and 2.9 (2.4 to 3.6), $\mathrm{p}<0.01$, for ACM (figure 3). 


\begin{tabular}{|c|c|c|c|c|c|c|}
\hline \multirow{2}{*}{$\begin{array}{l}\text { Outcome } \\
\text { Index eGFR category, } \mathrm{mL} / \mathrm{min} / 1.73 \mathrm{~m}^{2}\end{array}$} & \multirow{2}{*}{$\begin{array}{l}\text { Patients with } \\
\text { event, } \mathbf{n}(\%)\end{array}$} & \multicolumn{5}{|c|}{$\mathrm{HR}(95 \% \mathrm{Cl})$} \\
\hline & & \multirow[t]{2}{*}{ Crude model } & \multirow[t]{2}{*}{ Multivariable model* } & \multicolumn{2}{|c|}{ Multivariable model* } & \multirow[t]{2}{*}{ p-value } \\
\hline \multicolumn{4}{|l|}{$\mathrm{CV}$ death } & & & \\
\hline$<15$ & $55(10.1)$ & $8.0(6.0,10.5)^{\dagger}$ & $2.9(2.2,3.9)^{\dagger}$ & & $\longmapsto$ & $<0.01$ \\
\hline$\geq 15$ to $<30$ & $107(8.6)$ & $6.1(5.0,7.5)^{\dagger}$ & $2.3(1.8,2.8)^{\dagger}$ & & $\mapsto-1$ & $<0.01$ \\
\hline$\geq 30$ to $<60$ & $452(3.2)$ & $1.8(1.6,2.1)^{\dagger}$ & $1.1(0.9,1.2)$ & 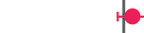 & & 0.27 \\
\hline$\geq 60$ to $<90$ (reference) & $551(1.8)$ & 1.0 & 1.0 & $\phi$ & & \\
\hline$\geq 90$ to $<120$ & $192(2.2)$ & $1.2(1.1,1.5)^{\dagger}$ & $1.6(1.4,1.9)^{\dagger}$ & & $1-1$ & $<0.01$ \\
\hline$\geq 120$ to $\leq 200$ & $54(3.7)$ & $2.4(1.9,3.2)^{\dagger}$ & $2.6(2.0,3.5)^{\dagger}$ & & $\longmapsto$ & $<0.01$ \\
\hline \multicolumn{7}{|l|}{ All-cause mortality $\ddagger$} \\
\hline$<15$ & 70 (12.9) & $5.9(4.6,7.5)^{\dagger}$ & $2.7(2.1,3.5)^{\dagger}$ & & $\longmapsto$ & $<0.01$ \\
\hline$\geq 15$ to $<30$ & $136(10.9)$ & $4.5(3.8,5.4)^{\dagger}$ & $2.1(1.8,2.6)^{\dagger}$ & & $\mapsto-1$ & $<0.01$ \\
\hline$\geq 30$ to $<60$ & $666(4.7)$ & $1.6(1.4,1.7)$ & $1.0(0.9,1.2)$ & ילי & & 0.43 \\
\hline$\geq 60$ to $<90$ (reference) & $958(3.1)$ & 1.0 & 1.0 & $\oint$ & & \\
\hline$\geq 90$ to $<120$ & $377(4.2)$ & $1.4(1.3,1.6)^{\dagger}$ & $1.7(1.5,1.9)^{\dagger}$ & & $\mapsto$ & $<0.01$ \\
\hline$\geq 120$ to $\leq 200$ & $113(7.8)$ & $3.0(2.4,3.6)^{\dagger}$ & $2.9(2.4,3.6)^{\dagger}$ & & $\mapsto$ & $<0.01$ \\
\hline \multicolumn{7}{|l|}{ All-cause hospitalisation } \\
\hline$<15$ & $294(54.2)$ & $4.3(3.9,4.9)^{\dagger}$ & $2.3(2.0,2.6)^{\dagger}$ & & r•r & $<0.01$ \\
\hline$\geq 15$ to $<30$ & $506(40.7)$ & $2.4(2.2,2.7)^{\dagger}$ & $1.5(1.3,1.6)^{\dagger}$ & & r| & $<0.01$ \\
\hline$\geq 30$ to $<60$ & $4363(30.4)$ & $1.4(1.4,1.5)^{\dagger}$ & $1.1(1.0,1.1)$ & $p$ & & $<0.01$ \\
\hline$\geq 60$ to $<90$ (reference) & $7266(23.5)$ & 1.0 & 1.0 & $\phi$ & & \\
\hline$\geq 90$ to $<120$ & $2189(24.5)$ & $1.1(1.0,1.1)^{\dagger}$ & $1.2(1.2,1.3)^{\dagger}$ & & 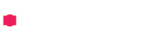 & $<0.01$ \\
\hline$\geq 120$ to $\leq 200$ & $485(33.4)$ & $1.8(1.7,2.0)^{\dagger}$ & $2.2(2.0,2.4)^{\dagger}$ & & 바 & $<0.01$ \\
\hline \multicolumn{7}{|l|}{ Any $\mathrm{CV}$ event§ } \\
\hline$<15$ & $257(47.4)$ & $7.2(6.4,8.2)^{\dagger}$ & $3.2(2.8,3.6)^{\dagger}$ & & $1 \bullet-1$ & $<0.01$ \\
\hline$\geq 15$ to $<30$ & $414(33.3)$ & $3.8(3.4,4.2)^{\dagger}$ & $1.7(1.5,1.9)^{\dagger}$ & & r.r & $<0.01$ \\
\hline$\geq 30$ to $<60$ & $3037(21.2)$ & $1.8(1.7,1.9)^{\dagger}$ & $1.1(1.1,1.2)^{\dagger}$ & e & & $<0.01$ \\
\hline$\geq 60$ to $<90$ (reference) & $3998(12.9)$ & 1.0 & 1.0 & $\phi$ & & \\
\hline$\geq 90$ to $<120$ & $1040(11.6)$ & $0.9(0.9,1.0)$ & $1.2(1.2,1.3)^{\dagger}$ & & 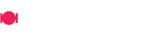 & $<0.01$ \\
\hline$\geq 120$ to $\leq 200$ & $181(12.5)$ & $1.2(1.0,1.3)^{\dagger}$ & $1.9(1.6,2.2)^{\dagger}$ & & $\mapsto-1$ & $<0.01$ \\
\hline \multicolumn{7}{|l|}{ Any kidney event } \\
\hline$<15$ & $185(34.1)$ & $280.0(216.0,364.0)^{\dagger}$ & $204.0(151.0,276.0)^{\dagger}$ & ** & & $<0.01$ \\
\hline$\geq 15$ to $<30$ & $120(9.7)$ & $50.4(38.1,66.8)^{\dagger}$ & $37.1(27.5,50.1)^{\dagger}$ & ** & & $<0.01$ \\
\hline$\geq 30$ to $<60$ & $118(0.8)$ & $3.3(2.5,4.3)^{\dagger}$ & $2.8(2.1,3.7)^{\dagger}$ & & $\longmapsto$ & $<0.01$ \\
\hline$\geq 60$ to $<90$ (reference) & $82(0.3)$ & 1.0 & 1.0 & $\phi$ & & \\
\hline$\geq 90$ to $<120$ & $19(0.2)$ & $0.8(0.5,1.4)$ & $0.9(0.6,1.5)$ & $\longmapsto$ & & 0.74 \\
\hline$\geq 120$ to $\leq 200$ & $3(0.2)$ & $0.9(0.3,3.0)$ & $1.1(0.4,3.6)$ & & $\longrightarrow$ & 0.82 \\
\hline & & & ${ }_{0.25}^{1}$ & 0.50 & 2.00 & \\
\hline
\end{tabular}

Figure 3 Risk of CV, mortality, hospitalisation and kidney outcomes by index eGFR category. ${ }^{*}$ Cox proportional hazard model for time-to-event, adjusted for age, sex, medical history, baseline type 2 diabetes status, baseline $\mathrm{ECl}$ score and baseline medication use. Categories 2-6 (index eGFR values of $<15, \geq 15$ to $<30, \geq 30$ to $<60, \geq 90$ to $<120, \geq 120$ to $\leq 200$ ) were analysed by outcome, with index eGFR Category 1 (index eGFR of $\geq 60$ to $<90$ ) as reference. †Statistically significant HRs. $\ddagger$ Based on inpatient death data. §Stroke, chronic heart failure or myocardial infarction. ๆEnd-stage kidney disease, dialysis, acute kidney failure or kidney transplant. ${ }^{*}$ These HRs are not presented in the forest plot, as they are extremely high values and so would distort the other HRs in the plot. CV, cardiovascular; ECl, Elixhauser Comorbidity Index; eGFR, estimated glomerular filtration rate; HR, hazard ratio.

Below-normal eGFR categories showed an excess risk for any kidney event (HR $(95 \% \mathrm{CI})$ for the $<15 \mathrm{~mL}$ / $\min / 1.73 \mathrm{~m}^{2}$ category: 204.0 (151.0 to 276.0 ), $\mathrm{p}<0.01$; and for the $\geq 15$ to $<30 \mathrm{~mL} / \mathrm{min} / 1.73 \mathrm{~m}^{2}$ category: 37.1 (27.5 to 50.1$), \mathrm{p}<0.01)$, whereas risk in the abovenormal eGFR categories was comparable with the reference group (figure 3 ).

Furthermore, we found that when increasing 1 unit $\left(\mathrm{mL} / \mathrm{min} / 1.73 \mathrm{~m}^{2}\right)$ of eGFR, the risk of any $\mathrm{CV}$ event was reduced by approximately $1 \%$ and any kidney event by $8 \%$ (data not shown).

\section{Interaction between index eGFR and eGFR slope}

In sensitivity analyses, we found that the addition of covariates increased the risk in eGFRinc slope categories of any kidney event from non-significant (HR $(95 \%$ CI) 0.7 (0.5 to 1.0$)$ for the $>1$ to $\leq 3 \mathrm{~mL} / \mathrm{min} / 1.73 \mathrm{~m}^{2}$ category and $1.0(0.6$ to 1.7$)$ in the $>3 \mathrm{~mL} / \mathrm{min} / 1.73 \mathrm{~m}^{2}$ category) to significant (1.6 (1.1 to 2.5$)$ and 3.6 (2.2 to 5.9 ), respectively) (figure 2). Using stepwise selection of covariates, we found that index eGFR had a particularly notable impact on the increase in risk between the two models, which led us to our further investigate the relationship between eGFR slope and index eGFR and to subsequently develop the 'full model', which included the interaction term.

The addition of the interaction term for eGFR slope and index eGFR appeared to reduce the association of increasing slopes and clinical outcomes. For instance, the HRs for eGFRinc slope groups for any kidney event were reduced from $>1$ and significant, to $<1$ and nonsignificant between the multivariable and full models: the HR $(95 \% \mathrm{CI})$ for category 5 ( $>1$ to $\leq 3 \mathrm{~mL} / \mathrm{min} / 1.73$ $\mathrm{m}^{2} /$ year) versus the reference category was 1.6 (1.1 to 2.5 ) in the multivariable model (ie, without the interaction term) and 0.2 (0.0 to 0.6$)$ in the full model, and for category $6\left(>3 \mathrm{~mL} / \mathrm{min} / 1.73 \mathrm{~m}^{2} /\right.$ year $), 3.6$ (2.2 to 5.9 ) and 0.5 (0.1 to 2.2), respectively. Therefore, for any kidney event, addition of the interaction term led to a change from increased risk versus the reference group to reduced risk in the eGFRinc groups. The difference in the risk of clinical outcomes between the crude/multivariable models and the full model among 
the eGFR slope groups, as shown in figure 2, demonstrates the notable impact of the index eGFR and eGFR slope interaction term on the association between eGFR slopes and risk of clinical outcomes, as the crude model and multivariable models do not include the interaction term.

\section{Subgroup analyses by T2D and tumour at baseline}

The study population included 21817 (38\%) patients with T2D and $24980(43 \%)$ patients with tumour at baseline. Online supplemental table 1 presents the baseline demographics for the subgroups with and without T2D as well as the subgroups with and without history of tumour. More patients in the subgroups with T2D and without tumour subgroups had comorbidities such as hyperlipidaemia, hypertension and CV disease at baseline compared with the subgroups without T2D and with tumour. Furthermore, more patients in the subgroups with T2D and without tumours used antihypertensive and antiplatelet medications, calcium channel blockers and renin-angiotensin-aldosterone system inhibitors at baseline compared with the subgroups without T2D and with tumours.

Stratified subgroup analyses of this study population indicated that in patients with a history of T2D and without a history of tumours at baseline, eGFRdec groups experienced a significantly increased risk of any kidney event compared with the reference group: HR (95\% CI) 2.1 (1.1 to 4.3) and 2.6 (1.3 to 5.2) for the $\leq-5$ and $>-5$ to $\leq-3 \mathrm{~mL} / \mathrm{min} / 1.73 \mathrm{~m}^{2} /$ year groups, respectively, in the subgroup with $\mathrm{T} 2 \mathrm{D} ; 2.3$ (1.3, to 4.2 ) and 3.1 (1.7 to 5.6), respectively, in the subgroup without tumours. In contrast, the subgroups without T2D and with tumours showed a trend of reduced risk of any kidney event in the rapid eGFRdec group compared with the reference group: 0.9 (0.4 to 1.8$)$ in the subgroup without T2D; 0.5 (0.2 to 1.3$)$ in the subgroup with tumours (online supplemental table 2).

Furthermore, in the rapid eGFRinc groups, a significantly increased risk of ACM was observed in the subgroups without T2D and with tumours compared with the reference group: HR (95\% CI) 2.4 (1.3 to 4.3) for the subgroup without T2D and 1.8 (1.1 to 3.2) for the subgroup with tumours. In contrast, a moderately reduced risk compared with the reference group was suggested in the subgroups with T2D or without tumours: HR (95\% CI) 0.7 (0.4 to 1.5) for the subgroup with T2D; 0.9 (0.4 to 2.1) for the subgroup without tumours (online supplemental table 2). Similarly, in the rapid eGFRdec groups, a large increase in risk of ACM compared with the reference group was observed in the subgroups without T2D and with tumours (HR (95\% CI) 3.4 (2.0 to 5.9) and 3.4 (2.1 to 5.6), respectively) compared with a moderately increased risk in the subgroups with T2D and without tumour (2.0 (1.1 to 3.8 ) and 2.3 (1.0 to 5.0), respectively) (online supplemental table 2).

\section{DISCUSSION}

This study found that in an observational Japanese cohort, not only declining eGFR slopes and belownormal eGFR indicate an increased risk for poorer clinical outcomes but increasing eGFR slopes and above-normal eGFR might also be relevant indicators of an increased risk for certain adverse clinical outcomes. In general, declining eGFR slopes were associated with poorer clinical outcomes in comparison with those who experienced little-to-no change in eGFR over time. Interestingly, a trend towards poorer clinical outcomes with increasing eGFR slopes was also suggested, though statistical significance was not reached. We also found that risk of mortality, hospitalisation and $\mathrm{CV}$ outcomes were not only increased with below-normal eGFR values but also with above-normal eGFR; however, the risk of kidney outcomes was only increased in below-normal eGFR ranges.

The association between declining eGFR slopes and increased mortality risk demonstrated in this study is in line with existing evidence in non-Japanese cohorts. ${ }^{19-21}$ In a 2014 meta-analysis by the CKD Prognosis Consortium, there was an increased risk of end-stage kidney disease with declining eGFR slope: HR (95\% CI) for an eGFR change of $-57 \%$ over 2 years of 32.1 (22.3 to 46.3 ) and 57.2 (21.9 to 149.1) for patients with baseline eGFR $<60 \mathrm{~mL} / \mathrm{min} / 1.73 \mathrm{~m}^{2}$ and $\geq 60 \mathrm{~mL} / \mathrm{min} / 1.73$ $\mathrm{m}^{2}$, respectively. ${ }^{21}$ This supports our finding that there was an association between declining eGFR slopes and adverse kidney outcomes. Likewise, a 2018 study by Van Pottelbergh et al found that declining eGFR slopes were associated with poorer CV outcomes, ${ }^{11}$ and a 2020 analysis of Chronic Renal Insufficiency Cohort study participants found increased $\mathrm{CV}$ and mortality risk with declining eGFR slopes in patients with CKD. ${ }^{3}$

Although it is widely accepted that declining eGFR slopes are a predictor for poor clinical outcomes, our data suggest that increasing slopes might also have clinical relevance regarding the risk for poor clinical outcomes. In a study of patients with CKD reported in 2011, the adjusted HR for mortality in the upper (increasing) eGFR slope tertiles relative to the middle (stable) tertile was $1.42(95 \%$ CI 1.20 to 1.69$) .{ }^{20} \mathrm{~A}$ similar association between increasing eGFR slope and mortality was indicated in a 2013 study, which observed that an eGFR increase in $\geq 5 \mathrm{~mL} / \mathrm{min} / 1.73 \mathrm{~m}^{2} /$ year was significantly associated with an increased risk of mortality (HR (95\% CI) 2.20 (2.10 to 2.31$)$ ) in patients with and without CKD. ${ }^{19}$ In patients with established CKD, typically defined as eGFR $<60 \mathrm{~mL} / \mathrm{min} / 1.73 \mathrm{~m}^{2}$, declining eGFR slopes have been indicated as having predictive value of clinical outcomes ${ }^{3} 12$ 21-24; however, the present study indicates that eGFR slopes might also be of value in evaluating clinical risk in patients with normal or above-normal eGFR.

Our data suggest a higher risk of ACM in both eGFRinc and eGFRdec groups, which might be attributable to different underlying comorbidities in these 
groups versus the reference group. In the rapid eGFRdec group $\left(\leq-5 \mathrm{~mL} / \mathrm{min} / 1.73 \mathrm{~m}^{2} /\right.$ year $)$, the association with increased mortality may be due to progression of kidney impairment and the known related risk of $\mathrm{CV}$ events ${ }^{3}$; whereas, in the rapid eGFRinc group (>3 $\mathrm{mL} / \mathrm{min} / 1.73 \mathrm{~m}^{2} /$ year), the association with increased mortality, though non-significant, might be related to other predominating comorbidities, such as higher incidence of tumours compared with the reference group. Increases in eGFR could also indicate the presence of other relevant pathologies, such as muscle wasting, haemodilution, kidney-related hyperfiltration or recovery from AKI, which cannot be differentiated based on the data available in this study.

In this study, we found that both above-normal and below-normal index eGFR ranges were associated with a higher risk of CV death, ACM, ACH and any CV event clinical outcomes. However, only below-normal index eGFR values were shown to be associated with poorer kidney outcomes. Our findings suggest that index eGFR might play a major role regarding the risk of $\mathrm{CV}$ and kidney events. A wealth of evidence supports our finding that below-normal eGFR is associated with increased risk of poor clinical outcomes ${ }^{12526}$; however, much less is known about above-normal eGFR and risk of clinical outcomes.

In the current analysis, we found an interaction effect between index eGFR and eGFR slopes. Comparison of the HRs produced using the crude and multivariable models versus the full model suggests that the association between eGFR slopes and the measured outcomes may have been partially mediated by the interaction effect between eGFR slope and index eGFR. In eGFRinc groups, higher index eGFR values increased the risk of any kidney event by approximately $3 \%-4 \%$, and in the eGFRdec groups, while higher index eGFR reduced the risk of any kidney event (data not shown). Therefore, our data suggest that increasing slopes are not always predictive of a reduction in the risk of adverse clinical outcomes and should be considered in conjunction with baseline eGFR value in order to predict clinical risk. There is currently a lack of understanding regarding the need to interpret eGFR slope data in relation to baseline eGFR; to our knowledge, previous publications have not investigated the interaction effect between eGFR slope and baseline eGFR. Based on our study results, adding the interaction term of eGFR slope and index eGFR has a notable impact on the risk of adverse clinical outcomes, highlighting the importance of interpreting the risk of outcomes based on eGFR slope data in the context of baseline eGFR values.

These findings suggest that the use of eGFR slopes could add value in clinical practice by supporting patient risk stratification for adverse clinical outcomes. Interestingly, increasing eGFR slopes and above-normal eGFR levels might be indicative of increased risk for adverse clinical outcomes in certain patient groups (ie, they do not necessarily reflect a true improvement in kidney function) and should, therefore, be interpreted with caution. The development of tools that enable clinicians to derive yearly kidney function decline from available patient laboratory data histories could help to foster uptake of eGFR slope use in clinical practice. Further investigations are warranted in order to provide a more granular understanding of the clinical relevance of eGFR slopes across the full range of kidney function.

\section{Strengths}

This study included a large number of patients with eGFR data gathered in a real-world setting, which was not restricted to a specific high-risk patient population. In addition, the association of both eGFR slope and eGFR index categories with several important clinical outcomes was determined using multiple statistical models, which is supportive of the robustness of the risk estimations. Due to the inclusion of the most recent data available in the MDV database, this study is likely to reflect the current patient population and, therefore, likely to be reliable and informative for use in guiding current clinical recommendations.

\section{Limitations}

The generalisability of our findings to the wider Japanese population outside of that included in the MDV database is unknown. For instance, the database includes a disproportionately high number of oncology patients, and the findings may apply only to this selected population. Additionally, inclusion of those with three or more eGFR values and $\geq 16$ years of age may have resulted in selection bias. Channelling bias with residual confounding may have led to imprecise estimates, because variables such as smoking, exercise and socioeconomic status, as well as other laboratory data which may impact the occurrence of CV outcomes, were not available. Furthermore, the use of claims diagnosis and procedure codes to define $\mathrm{CV}$ and mortality outcomes may have led to misclassification bias and imprecise estimates. We attempted to exclude patients with AKI at baseline from the study population; however, the use of diagnosis codes alone to identify AKI could have led to an underestimation of cases, as suggested by studies assessing the validity of ICD-10 codes. ${ }^{27-30}$ In addition, mortality data in this study were based on inpatient data only, which may have resulted in misclassification of the primary outcome. Another potential limitation of this study is that the use of only hospital-based data omits diagnoses and serum creatinine measurements taken in primary care settings. However, as the Japanese primary care system is not well-established, patients often visit secondary care services for primary care needs. ${ }^{31}$ As such, the impact of this limitation in the present study is expected to be modest in comparison with hospitalbased studies in countries with more established primary care systems. 


\section{CONCLUSION}

The results of this study in the Japanese population suggest that eGFR slope, in addition to absolute eGFR, may be of value in clinical practice for the identification of patients at high risk of adverse outcomes.

\section{Author affiliations}

${ }^{1}$ Real World Evidence Analytics Center of Excellence, Boehringer Ingelheim Corp USA, Ridgefield, Connecticut, USA

${ }^{2}$ Therapeutic Area CardioMetabolism Respiratory Medicine, Boehringer Ingelheim International GmbH, Ingelheim, Rheinland-Pfalz, Germany

${ }^{3}$ Vth Department of Medicine, University Medical Centre Mannheim, University of Heidelberg, Heidelberg, Baden-Württemberg, Germany

${ }^{4}$ Clinical Development and Medical Affairs, Nippon Boehringer Ingelheim, Tokyo, Japan

${ }^{5}$ Department of Nephrology, Hospital rechts der Isar, Faculty of Medicine, Technical University Munich, Munich, Germany

${ }^{6}$ Medicine Division, Nippon Boehringer Ingelheim, Tokyo, Japan

${ }^{7}$ Department of Internal Medicine 1, Faculty of Medicine, Shimane University,

Shimane, Japan

${ }^{8}$ Division of Anticipatory Molecular Food Science and Technology, Medical Research Institute, Kanazawa Medical University, Kahoku-gun, Ishikawa, Japan

Acknowledgements Medical writing assistance, supported financially by Boehringer Ingelheim, was provided by Sally Neath of Elevate Scientific Solutions during the preparation of this article. The authors were fully responsible for all content and editorial decisions, were involved at all stages of manuscript development and have approved the final version.

Contributors YO obtained the funding. YO, MHK and LZ developed the research design. $L Z$ is responsible for the overall content as the guarantor. $L Z$ analysed the data. LZ, SH, YO, MHK, DS, YN and KK interpreted the results, critically reviewed the manuscript and approved the final draft.

Funding This study was sponsored by Boehringer Ingelheim.

Competing interests LZ, SH, YO, DS and YN are employees of Boehringer Ingelheim. MHK was an employee of Boehringer Ingelheim at the time of the study. KK received lecture honoraria from Astellas, AstraZeneca, Boehringer Ingelheim, Dainippon-Sumitomo Pharma, Eli Lilly, Kyowa-Hakko Kirin, Mitsubishi-Tanabe, MSD, Novo Nordisk, Ono, Sanofi, and Taisho-Toyama; scholarship grants from Boehringer Ingelheim (Japan), Chugai, Kowa, Mitsubishi-Tanabe, and Ono; and joint research grants from Boehringer Ingelheim (Japan) and Taisho-Toyama. Boehringer Ingelheim (Japan), Kyowa-Hakko Kirin, Mitsubishi-Tanabe, Ono, and Taisho-Toyama contributed to establishing the Division of Anticipatory Molecular Food Science and Technology, Medical Research Institute, Kanazawa Medical University.

Patient consent for publication Not applicable.

Ethics approval This study was approved by the Ethics Committee of Research Institute of Healthcare Data Science (number 2020008).

Provenance and peer review Not commissioned; externally peer reviewed.

Data availability statement We conducted a retrospective study using patient data from the MDV database, a commercially available database of health claims and administrative data from Japanese acute hospitals. Restrictions do apply to the availability of these data due to a contract between MDV and Boehringer Ingelheim and are thus unavailable to the public. For inquiries on the database analysed in this study, please contact MDV (https://en.mdv.co.jp).

Supplemental material This content has been supplied by the author(s). It has not been vetted by BMJ Publishing Group Limited (BMJ) and may not have been peer-reviewed. Any opinions or recommendations discussed are solely those of the author(s) and are not endorsed by BMJ. BMJ disclaims all liability and responsibility arising from any reliance placed on the content. Where the content includes any translated material, BMJ does not warrant the accuracy and reliability of the translations (including but not limited to local regulations, clinical guidelines, terminology, drug names and drug dosages), and is not responsible for any error and/or omissions arising from translation and adaptation or otherwise.

Open access This is an open access article distributed in accordance with the Creative Commons Attribution Non Commercial (CC BY-NC 4.0) license, which permits others to distribute, remix, adapt, build upon this work non-commercially, and license their derivative works on different terms, provided the original work is properly cited, appropriate credit is given, any changes made indicated, and the use is non-commercial. See: http://creativecommons.org/licenses/by-nc/4.0/.

\section{ORCID iDs}

Ling Zhang http://orcid.org/0000-0003-2472-9544

Keizo Kanasaki http://orcid.org/0000-0002-9563-502X

\section{REFERENCES}

1 Chronic Kidney Disease Prognosis Consortium, Matsushita K, van der Velde M, et al. Association of estimated glomerular filtration rate and albuminuria with all-cause and cardiovascular mortality in general population cohorts: a collaborative meta-analysis. Lancet 2010;375:2073-81.

2 Gansevoort RT, Matsushita K, van der Velde M, et al. Lower estimated GFR and higher albuminuria are associated with adverse kidney outcomes. A collaborative meta-analysis of general and highrisk population cohorts. Kidney Int 2011;80:93-104.

3 Orlandi PF, Xie D, Yang W, et al. Slope of kidney function and its association with longitudinal mortality and cardiovascular disease among individuals with CKD. J Am Soc Nephrol 2020;31:2912-23.

4 Grams ME, Sang Y, Ballew SH, et al. Evaluating glomerular filtration rate slope as a surrogate end point for ESKD in clinical trials: an individual participant meta-analysis of observational data. J Am Soc Nephrol 2019;30:1746-55.

5 Inker LA, Heerspink HJL, Tighiouart H, et al. Gfr slope as a surrogate end point for kidney disease progression in clinical trials: a metaanalysis of treatment effects of randomized controlled trials. J Am Soc Nephrol 2019;30:1735-45.

6 Levey AS, Gansevoort RT, Coresh J, et al. Change in albuminuria and GFR as end points for clinical trials in early stages of CKD: a scientific workshop sponsored by the National kidney Foundation in collaboration with the US food and drug administration and European medicines Agency. Am J Kidney Dis 2020;75:84-104.

7 Torres VE, Higashihara E, Devuyst O, et al. Effect of Tolvaptan in autosomal dominant polycystic kidney disease by CKD stage: results from the tempo 3:4 trial. Clin J Am Soc Nephrol 2016;11:803-11.

8 Torres VE, Chapman AB, Devuyst O, et al. Tolvaptan in Later-Stage autosomal dominant polycystic kidney disease. $N$ Engl J Med 2017;377:1930-42

9 Oshima M, Jun M, Ohkuma T, et al. The relationship between eGFR slope and subsequent risk of vascular outcomes and all-cause mortality in type 2 diabetes: the ADVANCE-ON study. Diabetologia 2019;62:1988-97.

10 Nojima J, Meguro S, Ohkawa N, et al. One-Year eGFR decline rate is a good predictor of prognosis of renal failure in patients with type 2 diabetes. Proc Jpn Acad Ser B Phys Biol Sci 2017;93:746-54.

11 Van Pottelbergh G, Mamouris P, Opdeweegh N, et al. Is there a correlation between an eGFR slope measured over a 5-year period and incident cardiovascular events in the following 5 years among a Flemish general practice population: a retrospective cohort study. BMJ Open 2018;8:e023594.

12 Turin TC, Jun M, James MT, et al. Magnitude of rate of change in kidney function and future risk of cardiovascular events. Int J Cardiol 2016;202:657-65.

13 Perkovic V, Jardine MJ, Neal B, et al. Canagliflozin and renal outcomes in type 2 diabetes and nephropathy. $N$ Engl J Med 2019;380:2295-306.

14 Heerspink HJL, Stefansson BV, Chertow GM, et al. Rationale and protocol of the dapagliflozin and prevention of adverse outcomes in chronic kidney disease (DAPA-CKD) randomized controlled trial. Nephrol Dial Transplant 2020;35:274-82.

15 Brenner BM, Cooper ME, de Zeeuw D, et al. Effects of losartan on renal and cardiovascular outcomes in patients with type 2 diabetes and nephropathy. N Engl J Med 2001;345:861-9.

16 Lewis EJ, Hunsicker LG, Clarke WR, et al. Renoprotective effect of the angiotensin-receptor antagonist irbesartan in patients with nephropathy due to type 2 diabetes. $N$ Engl J Med Overseas Ed 2001;345:851-60.

17 Herrington WG, Preiss D, Haynes R, et al. The potential for improving cardio-renal outcomes by sodium-glucose co-transporter-2 inhibition in people with chronic kidney disease: a rationale for the EMPAKIDNEY study. Clin Kidney J 2018;11:749-61.

18 Bakris GL, Agarwal R, Anker SD, et al. Effect of Finerenone on chronic kidney disease outcomes in type 2 diabetes. $N$ Engl J Med Overseas Ed 2020;383:2219-29.

19 Turin TC, Coresh J, Tonelli M, et al. Change in the estimated glomerular filtration rate over time and risk of all-cause mortality. Kidney Int 2013;83:684-91. 
20 Perkins RM, Bucaloiu ID, Kirchner HL, et al. GFR decline and mortality risk among patients with chronic kidney disease. Clin J Am Soc Nephrol 2011;6:1879-86.

21 Coresh J, Turin TC, Matsushita K, et al. Decline in estimated glomerular filtration rate and subsequent risk of end-stage renal disease and mortality. JAMA 2014;311:2518-31.

22 Lambers Heerspink HJ, Tighiouart $\mathrm{H}$, Sang $\mathrm{Y}$, et al. GFR decline and subsequent risk of established kidney outcomes: a meta-analysis of 37 randomized controlled trials. Am J Kidney Dis 2014;64:860-6.

23 Al-Aly Z, Zeringue A, Fu J, et al. Rate of kidney function decline associates with mortality. J Am Soc Nephrol 2010;21:1961-9.

24 Naimark DMJ, Grams ME, Matsushita K, et al. Past decline versus current eGFR and subsequent mortality risk. J Am Soc Nephrol 2016;27:2456-66.

25 Go AS, Chertow GM, Fan D, et al. Chronic kidney disease and the risks of death, cardiovascular events, and hospitalization. $N$ Engl $J$ Med 2004;351:1296-305.

26 Eckardt K-U, Bansal N, Coresh J, et al. Improving the prognosis of patients with severely decreased glomerular filtration rate (CKD G4+): conclusions from a Kidney Disease: Improving Global Outcomes (KDIGO) Controversies Conference. Kidney Int 2018;93:1281-92.

27 Hwang YJ, Shariff SZ, Gandhi S, et al. Validity of the International Classification of Diseases, Tenth Revision code for acute kidney injury in elderly patients at presentation to the emergency department and at hospital admission. BMJ Open 2012;2:e001821.

28 Molnar AO, van Walraven C, McArthur E, et al. Validation of administrative database codes for acute kidney injury in kidney transplant recipients. Can J Kidney Health Dis 2016;3:108.

29 Ko S, Venkatesan S, Nand K, et al. International statistical classification of diseases and related health problems coding underestimates the incidence and prevalence of acute kidney injury and chronic kidney disease in general medical patients. Intern Med $J$ 2018;48:310-5.

30 Tomlinson LA, Riding AM, Payne RA, et al. The accuracy of diagnostic coding for acute kidney injury in England - a single centre study. BMC Nephrol 2013;14:58.

31 Kato D, Ryu H, Matsumoto T, et al. Building primary care in Japan: literature review. J Gen Fam Med 2019;20:170-9. 